\title{
Application of Carbon Fiber Ankle Foot Orthoses to Enhance Gait Outcomes for Individuals with Neurologic Gait Dysfunction
}

\begin{abstract}
Shearin SM*, Bauzaite E, Hall H and McCain KJ Department of Physical Therapy, The University of Texas Southwestern, USA

*Corresponding author: Shearin SM, Department of Physical Therapy, The University of Texas Southwestern, 5323 Harry Hines Blvd, Dallas, TX 75239-8876, USA
\end{abstract}

Received: September 06, 2017; Accepted: October 05, 2017; Published: October 12, 2017

\begin{abstract}
Introduction: There is evidence that ankle foot orthoses (AFO) designs can impact gait outcomes for patients with neurologic dysfunction. Few studies have examined the benefits of different materials such as CF orthoses compared to plastic orthoses. This is a critical need in view of the difference in expense and complexity of fabricating CF AFOs compared to more traditional, less expensive polypropylene devices.
\end{abstract}

Objective: The purpose of this study is to highlight excellent gait outcomes resulting from transitioning individuals from polypropylene AFOs and peroneal nerve stimulators to custom-fabricated CF AFOs in individuals with neurological impairments.

Materials and Methods: The participants included 13 individuals with neurologic gait dysfunction who were transitions from polypropylene AFOs to custom-fabricated CF orthoses. All of these individuals were experienced users of previous devices, either polypropylene or peroneal nerve stimulator. In order to highlight the effects of the orthoses while minimizing the effects of the physical therapy interventions, each participant received between 1 and 4 physical therapy sessions with the new orthoses before the data was collected.

Results: All 13 participants demonstrated improvements in their step length and gait velocity from initial to final condition. Many of the participants experienced improved step symmetry as well.

Conclusions: The participants in this study showed a positive trend for improvements in step length, step length differential, and gait velocity with the use of the CF AFOs. This clinical tool has the potential to significantly augment the treatment of these individuals and is an important avenue of future research.

Keywords: Gait; Orthosis; Brace; Carbon fiber; Neurologic rehabilitation

\section{Introduction}

Ankle-foot orthoses (AFOs) are often prescribed for persons with neurological conditions to improve their quality of gait. There are many different designs of ankle foot orthoses as well as different materials that are utilized. Orthoses can be designed topromote dorsiflexion assistance, aid in knee stabilization, improve foot positioning and they may have the potential to enhance proprioceptive feedback. There is evidence in the literature that AFO designs can impact gait outcomes for patients with neurologic dysfunction. AFOs have been shown to increase walking speed, decrease energy expenditure, and positively impact stance phase as well as foot position [1-4]. Miyazaki also suggested that individual AFO design could significantly impact gait mechanicsas well as muscle activation secondary tothe external input afforded by the device [5]. Some AFO designs can supplement plantarflexion strength which provides critical stability in mid to late stance $[5,6]$.

Currently, there are two commonly used types of bracing material, polypropylene (plastic) and carbon fiber (CF) as well as some commonly utilized orthosis designs. Polypropylene is a widely used material that is generally easy to work with and it is relatively inexpensive. Polypropylene has historically been used to fabricate posterior leaf spring, solid ankle, and double action AFOs. A posterior leaf spring AFO is effective for reducing foot drop/drag during swing phase and allows for some plantar flexion at loading response, but is not ideal for persons with posterior compartment weakness due to the lack of anterior stability [7]. Double action AFOs have been found to not only assist with dorsiflexion during swing, but also to allow for plantar flexion during loading response and even to assist in regaining plantarflexion strength after stroke [4]. Solid ankle AFOs, while still widely used, do function to aid dorsiflexion during swing but interfere with normal loading response by limiting plantar flexion. They also serve to limit tibial translation during stance, which interferes with a trailing limb and typical muscle activation (REF).

In contrast, CFis a less familiar material to orthotists and is more expensive than polypropylene. CF is thought to besuperior to polypropylene due to its energy storing capacity and lightweight quality. CF has been used to fabricate similar designs as polypropylene, such as the posterior leaf spring and solid ankle. There is evidence in the literature of using CF orthoses in persons with neurologic diagnoses. 
Table 1: Patient Demographics.

\begin{tabular}{|c|c|c|c|c|c|}
\hline Patient Number & Diagnosis & Years since diagnosis & Age & Gender & Number of Treatments \\
\hline 1 & Multiple Sclerosis & 6 & 54 & $\mathrm{~F}$ & 3 \\
\hline 2 & Stroke & 5 & 73 & $\mathrm{~F}$ & 4 \\
\hline 3 & Transverse myelitis & 16 & 48 & $\mathrm{~F}$ & 4 \\
\hline 4 & Multiple sclerosis & 23 & 51 & $\mathrm{~F}$ & 2 \\
\hline 5 & Spinal cord injury & 5 & 23 & M & 1 \\
\hline 6 & Arteriovenous malformation & 3 & 19 & M & \\
\hline 7 & Stroke & 3 & 64 & M & 3 \\
\hline 8 & Stroke & 2 & 51 & M & 1 \\
\hline 9 & Foot drop post L4-L5 and L5-S1 laminectomy & 1 & 76 & M & 1 \\
\hline 10 & Multiple sclerosis & 30 & 69 & $\mathrm{~F}$ & 1 \\
\hline 11 & Stroke & 2 & 62 & $\mathrm{~F}$ & 3 \\
\hline 13 & Multiple sclerosis & 13 & 44 & M & 1 \\
\hline
\end{tabular}

Table 2: Orthoses and Assistive Devices.

\begin{tabular}{|c|c|c|c|c|}
\hline Patient Number & Previous bracing & Unilateral/bilateral CF orthosis & Initial assistive device & Final assistive device \\
\hline 1 & Peroneal nerve stimulator & Right & Single tip cane & Bilateral forearm crutches \\
\hline 2 & Tamarack with a plantarflexion stop & Right & Walker & No change \\
\hline 3 & Polypropylene & Unilateral & Single tip cane & Forearm crutch \\
\hline 4 & Polypropylene & Unilateral & Cane & No change \\
\hline 5 & Double action & Right & Single tip cane & No change \\
\hline 6 & Double action & Right & None & No change \\
\hline 7 & Double action & Right & Bilateral forearm crutches & No change \\
\hline 8 & Double action & Right & Single tip cane & No change \\
\hline 9 & Polypropylene & Unilateral & Cane occasionally & No change \\
\hline 10 & Polypropylene & Bilateral & Walker & No change \\
\hline 11 & Polypropylene & Unilateral & None & No change \\
\hline 13 & Unilateral Double action & Bilateral & Unilateral forearm crutch & No change \\
\hline
\end{tabular}

Bartonek et al. determined that there was a positive energy storing effect of posterior leaf spring CF orthoses in persons with neurologic diagnoses [8]. Bregman et al determined that CFAFOs have energy conservation properties (Bregman, Harlaar et al. 2012). Additionally, CF designs typically have higher rates of overall satisfaction because of their more modern appearance [9].

Few studies have examined the benefits of CF orthoses compared to plastic orthoses. When assessed collectively, studies show that orthoses overall positively impacts gait in persons with neurologic injury, but clear clinical guidelines are unavailable due to the diversity of options studied and the lack of comparative studies. This is a critical need in view of the difference in expense and complexity of fabricating CF AFOs compared to more traditional, less expensive polypropylene devices. The current study is intended to add to the current body of knowledge in this area. The purpose of this study is to highlight excellent gait outcomes resulting from transitioning individuals from polypropylene AFOs and peroneal nerve stimulators to customfabricated CF AFOs in individuals with neurological impairments.

\section{Case Description}

\section{Participants}

The Institutional Review Board approved this retrospective data collection. The principle and co-investigators identified individuals from the School of Health Professions Neurologic Physical Therapy Gait Disorders Clinic with neurologic diagnoses who were treated with custom-fabricated CF ankle foot orthoses (unilateral or bilateral). The participants were retrospectively selected if their device was changed from a non-CF brace or peroneal nerve stimulator to a customfabricated CF orthosis. The principle and co-investigators reviewed the electronic patient physical therapy records from the School of Health Professions Neurologic Physical Therapy Gait Disorders Clinic to collect the data which was de-identified.

The group of participants included 13 individuals who were treated with custom-fabricated CF bracing and all had gait dysfunction. The individuals ranged in age from 19-76 years old. The participants' diagnosis, years since diagnosis, age, gender, and number of treatments after orthosis fitting to data collection are listed in Table 


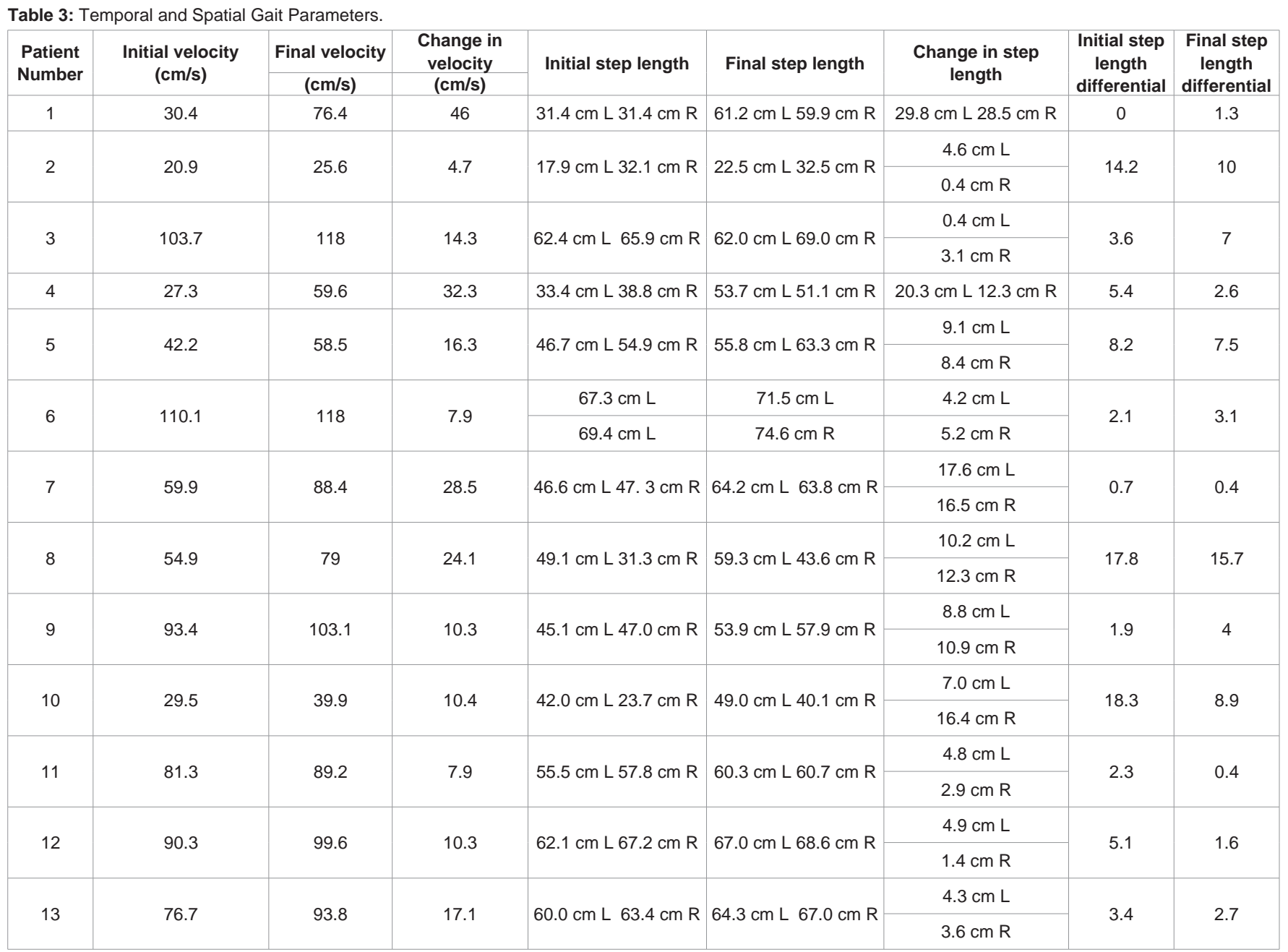

Cm: Centimeter; s: second; L: left; R: right.

Initial testing in original orthosis(es), Final testing in custom carbon fiber orthosis(es).

1. Table 2 details the participants' previous bracing (polypropylene or peroneal nerve stimulator), current CF bracing (unilateral or bilateral), as well as their initial and final assistive device use. All of these individuals were experienced users of previous devices, either polypropylene or peroneal nerve stimulator (> 6 months duration).

\section{Interventions}

The purpose of this study was to highlight the impact of customfabricated CF ankle foot orthoses application on individuals living with neurologic dysfunction. Each of the individuals in this study changed theorthoses they were accustomed to using (polypropylene and peroneal nerve stimulators) to custom-fabricated CF bracing. The CF orthoses were all fabricated in the same facility in Dallas, Texas with a single posterior leaf spring design. In order to highlight the effects of the orthoses while minimizing the effects of the physical therapy interventions, each participant received between 1 and 4 physical therapy sessions with the new orthoses before the data was collected. The gait interventions were similar for all participants despite diagnosis and included education for use of orthoses for anterior tibial support, focus on symmetry with the use of assistive devices or external cues such as tape on the floor, metronome, or laser beam, and education on strengthening and stretching for both the home and clinic as warranted per individual.The aim of gait training was to set the environment for practice with optimal gait mechanics.

\section{Measures and Methods}

All participants were under the supervision and the testing was performed by a licensed physical therapist in the Gait Disorders Clinic within the David M. Crowley RehabilitationResearch Laboratory at the University of Texas Southwestern Medical Center, Dallas, TX. All statistical analyses will be performed using SPSS version 25.0 for Windows (SPSS, Inc. Chicago, IL.). The primary dependent variables analysis will be performed bya paired t-test. Significance will be set at $\mathrm{p}<.05$ for all analysis.

\section{Gait temporal and spatial parameters (GAITRite)}

For the purpose of this study, the GAITRite ${ }^{\text {TM }}$ computerized gait analysis system (CIR Systems, Havertown, PA, USA) was used to collect data on gait parameters including gait velocity and step length. This system has been demonstrated to be reliable and valid with individuals with neurologic gait dysfunction [10-12]. The system consists of a 14-foot pressure-sensitive mat with an area of activation measuring 24 inches $(61 \mathrm{~cm})$ wide and 192 in $(487 \mathrm{~cm})$ long with a total of 18,432 sensors. Data was sampled at $1000 \mathrm{~Hz}$. Participants 
Table 4: Statistical Analysis.

Table 4: Statistical Analysis.
\begin{tabular}{|c|c|c|}
\hline Variable & $\begin{array}{c}\text { Initial mean }( \pm \text { standard } \\
\text { deviation) }\end{array}$ & $\begin{array}{c}\text { Final mean }( \pm \text { standard } \\
\text { deviation) }\end{array}$ \\
\hline Velocity & $63.1(31.4)$ & $80.7(28.3)^{\star}$ \\
\hline Step length right & $48.5(15.8)$ & $57.9(12.5)^{\star}$ \\
\hline Step length left & $47.7(14.2)$ & $57.3(12.1)^{\star}$ \\
\hline $\begin{array}{c}\text { Step length } \\
\text { differential }\end{array}$ & $6.4(6.4)$ & $5.0(4.5)^{*}$ \\
\hline
\end{tabular}

*Statistically different compared to initial values, $p<.001$.

walked over the walkway a total of six to eight times at their selfselected walking velocity. Data was collected initially with original orthoses and finally with custom-fabricated CF orthoses within 4 physical therapy sessions of fitting with the CF orthoses.

\section{Results}

The GAITRite ${ }^{\mathrm{TM}}$ was used to measure temporal and spatial gait parameters (Table 3). All 13 participants' demonstrated statistically significant improvements in their step length, step length differential, gait velocity from the initial condition in their original orthoses to final condition in the CF orthoses (Table 4 for means, standard deviation, and statistical differences). Only two of the participants had a change in their assistive devices during the intervention period.

\section{Discussion}

The purpose of this retrospective study was to describe the impact of custom-fabricated CF bracing on 13 individuals who were previously braced in traditional polypropylene orthoses or peroneal nerve stimulators. The primary outcomes were step length, gait velocity, and step length differential as recorded by a GAITRite ${ }^{\text {TM }}$ mat. The participants in this study showed statistically significant improvements in step length, step length differential, and gait velocity with the use of the CF AFOs. In this cohort, 100 percent of the participants improved their step length and gait velocity, while 69 percent decreased their step length differential. These changes likely highlight the effects of the orthoses on gaitas there was not substantial time for motor learning considering the small number of physical therapy interventions (less than 4).

There is support in the literature for the use of AFOs for individuals with neurologic conditions. However, there is very little literature comparing CF to other forms of orthoses. CF orthoses provide more stance support and energy storing during terminal stance compared to traditional polypropylene designs [13]. Further, CF braces provide energy during the third rocker which provides a forward propulsion effect [14]. In this cohort, improved stance support and forward propulsion may have resulted in longer step lengths and decreased step length differentials for most of the patients. Additionally, one large advantage to CF orthoses is the potential decrease in energy cost of walking in individuals with neurologic dysfunction $[15,16]$. The changes recorded in gait for this study may indicate improved gait efficiency especially regarding the improvements made in step length symmetry for many of the participants.

This is a heterogeneous group in terms of age, diagnosis, and yearspost diagnosis. Although this limits the ability to generalize the data, it also serves to highlight the successful use of custom-fabricated $\mathrm{CF}$ orthoses in a diverse patient population. It is encouraging to consider the excellent responses obtained in such a varied cohort. In addition, our experience with these devices is that individualsgenerally have a very positive perception of the custom-fabricated CF orthoses as they are lightweight, cosmetically appealing, and have smaller trim lines that make them cooler to wear.

Further research is needed to define best practice for the use of CF orthoses, custom-fabricated and off-the-shelf. Larger, randomized clinical trials are necessary to examine the impact of CF orthoses on gait kinematics for individuals with specific neurologic diagnoses. Further research can help to identifythe best candidates for CF bracing by allowing for longitudinal use of the devices, thus allowing for motor learning in the orthoses. There is currently a dearth of literature with regards to muscle activity in orthoses due in large part to study design $[17,18]$. However, there is potential muscle activity can be enhance with proper orthosis selection [4]. Finally, further research is needed to quantify the energy storing capabilities and impact of design and fabrication techniques on the effectiveness of CF orthoses.

\section{Conclusions}

This study reported the clinical results of transitioning individuals from traditional polypropylene orthoses and peroneal nerve stimulators into custom-fabricated CF-orthoses. Considering the profound number of people living with neurologic gait dysfunction, this area of research has the potential to impact a large range of individuals' functional mobility and quality of life. The individuals in this case study were trained with custom-fabricated CF AFOs that were designed to provide support and proprioceptive feedback which, improved gait speed, step length, and, for most, step length symmetry. This clinical tool has the potential to significantly augment the treatment of these individuals and is an important avenue of future research.

\section{References}

1. Abe H, Michimata A, Sugawara K, Sugaya N, Izumi S. Improving gait stability in stroke hemiplegic patients with a plastic ankle-foot orthosis. The Tohoku journal of experimental medicine. 2009; 218: 193-199.

2. Hesse S, Luecke D, Jahnke MT, Mauritz KH. Gait function in spastic hemiparetic patients walking barefoot, with firm shoes, and with anklefoot orthosis. International journal of rehabilitation research Internationale Zeitschrift fur Rehabilitations forschung Revue internationale de recherches de readaptation. 1996; 19: 133-141.

3. Mulroy SJ, Eberly VJ, Gronely JK, Weiss W, Newsam CJ. Effect of AFO design on walking after stroke: impact of ankle plantar flexion contracture. Prosthetics and orthotics international. 2010; 34: 277-292.

4. McCain KJ, Smith PS, Querry R. Ankle-foot orthosis selection to facilitate gait recovery in adults after stroke: a case series. JPO: Journal of Prosthetics and Orthotics. 2012; 24: 111-121.

5. Miyazaki S, Yamamoto S, Kubota T. Effect of ankle-foot orthosis on active ankle moment in patients with hemiparesis. Medical \& biological engineering \& computing. 1997; 35: 381-385.

6. Lehmann JF, Esselman PC, Ko MJ, Smith JC, deLateur BJ, Dralle AJ. Plastic ankle-foot orthoses: evaluation of function. Archives of physical medicine and rehabilitation. 1983; 64: 402-407.

7. May B, Lockard M. Prosthetics and orthotics in clinical practice: a case study approach. Philadelphia, PA: F A Davis; 2011; 256-257.

8. Bartonek A, Eriksson M, Gutierrez-Farewik EM. A new carbon fibre spring orthosis for children with plantarflexor weakness. Gait \& posture. 2007; 25: 652-656. 
9. Bartonek A, Eriksson M, Gutierrez-Farewik EM. Effects of carbon fibre spring orthoses on gait in ambulatory children with motor disorders and plantarflexor weakness. Developmental medicine and child neurology. 2007; 49: 615-620.

10. Nelson AJ, Zwick D, Brody S, Doran C, Pulver L, Rooz G, et al. The validity of the GaitRite and the Functional Ambulation Performance scoring system in the analysis of Parkinson gait. Neuro Rehabilitation. 2002; 17: 255-262.

11. Bilney B, Morris M, Webster K. Concurrent related validity of the GAITRite ${ }^{\circledR}$ walkway system for quantification of the spatial and temporal parameters of gait. Gait \& posture. 2003; 17: 68-74.

12. Wong JS, Jasani H, Poon V, Inness EL, Mcllroy WE, Mansfield A. Inter-and intra-rater reliability of the GAITRite system among individuals with sub-acute stroke. Gait \& posture. 2014; 40: 259-261.

13. Wolf SI, Alimusaj M, Rettig O, Döderlein L. Dynamic assist by carbon fiber spring AFOs for patients with myelomeningocele. Gait \& posture. 2008; 28 175-177.
14. Alimusaj M, Knie I, Wolf S, Fuchs A, Braatz F, Döderlein L. Functional impact of carbon fiber springs in ankle-foot orthoses. Der Orthopade. 2007; 36: 752756.

15. Bregman D, Harlaar J, Meskers C, De Groot V. Spring-like Ankle Foot Orthoses reduce the energy cost of walking by taking over ankle work. Gait \& posture. 2012; 35: 148-153.

16. Danielsson A, Sunnerhagen KS. Energy expenditure in stroke subjects walking with a carbon composite ankle foot orthosis. Journal of rehabilitation medicine. 2004; 36: 165-168.

17. Leung J, Moseley A. Impact of ankle-foot orthoses on gait and leg muscle activity in adults with hemiplegia: systematic literature review. Physiotherapy. 2003; 89: 39-55.

18. Tyson S, Sadeghi-Demneh E, Nester C. A systematic review and metaanalysis of the effect of an ankle-foot orthosis on gait biomechanics after stroke. Clinical rehabilitation. 2013; 27: 879-891.
Phys Med Rehabil Int - Volume 4 Issue 4 - 2017

ISSN : 2471-0377 | www.austinpublishing group.com

Shearin et al. (C) All rights are reserved
Citation: Shearin SM, Bauzaite E, Hall H and McCain KJ. Application of Carbon Fiber Ankle Foot Orthoses to Enhance Gait Outcomes for Individuals with Neurologic Gait Dysfunction. Phys Med Rehabil Int. 2017; 4(4): 1123. 